\title{
Wie beeinflusst die Feminisierung der Medizin die Medikalisierung weiblicher Biographien?
}

Im folgenden Artikel «Die Medikalisierung weiblicher Biographien im mittleren Alter» von Kolip und Glaeske wird von soziologisch-psychologischer Seite auf die Medikalisierung von weiblichen Umbruchphasen verwiesen: Schwangerschaft, Menopause. Die Autoren nehmen Bezug auf das "geschlechtsspezifisch diagnostizierte Krankheitsspektrum" und stellen kurz drei Hypothesen zur Erklärung geschlechtsspezifischer Krankheitsprävalenzen vor, um in der Folge vor allem auf die Verschreibung von Psychopharmaka an die Frauen einzugehen. Zwei Drittel aller sedierenden Psychopharmaka werden Frauen verabreicht durch in der Grundversorgung tätige Ärztinnen und Ärzte. Auffallend ist der Zusammenhang zwischen der fehlenden Erwerbstätigkeit vor allem ab dem 45. Altersjahr und dem Konsum von Beruhigungsmitteln.

Leider liefern die Autoren in dieser auch sonst etwas oberflächlichen Arbeit keine getrennten Daten zu den Verschreibegewohnheiten von Ärztinnen und Ärzten. Solche Daten wären von grossem Interesse im Hinblick auf die Entwicklung der Medizin, welche sich von einem Männer- zu einem Frauenberuf ändert: Im Jahr 2000 waren gemäss FMH-Mitgliederstatistik nur 16\% der Allgemeinmediziner und Internisten Frauen, einen entsprechenden Facharzttitel erwarben im selben Jahr jedoch zu $38 \%$ Ärztinnen. Diese Feminisierung der Medizin (vgl. auch Schweiz Ärztezeitung 2000;81:809 und 2001;82:1838-44) wird nebst sozioökonomischen Auswirkungen auf die Stellung des Berufes wohl auch zu einer Änderung der Interpretation von Lebensphasen, im speziellen der weiblichen Biographien, und vielleicht zu einer "Entmedikalisierung» führen. Weitere Arbeiten zu dieser Thematik sind zu erwarten - wir sind gespannt auf differenziertere Resultate!

Max Giger, Winterthur 